\title{
AN A PRIORI ESTIMATE FOR THE GAUSS CURVATURE OF NONPARAMETRIC SURFACES OF CONSTANT MEAN CURVATURE
}

\author{
JOEL SPRUCK ${ }^{1}$
}

\begin{abstract}
We consider surfaces of constant mean curvature in three-dimensional Euclidean space which have a nonparametric representation over a disc. It is shown that if the surface has a horizontal tangent plane at the origin of the disc, then the Gauss curvature of the surface at the origin satisfies an a priori bound. The existence of a bound is established by identifying and proving the existence of an extremal surface.
\end{abstract}

1. Introduction. We consider surfaces $S$ of constant mean curvature $H$ which have a nonparametric representation $z=u(x, y)$ over the disc $x^{2}+y^{2}<R^{2}$. This is equivalent to saying that $u(x, y)$ is a solution of the partial differential equation

$$
\left(1+u_{y}^{2}\right) u_{x x}-2 u_{x} u_{y} u_{x y}+\left(1+u_{x}^{2}\right) u_{y y}=2 H\left(1+u_{x}^{2}+u_{y}^{2}\right)^{3 / 2} .
$$

Without loss of generality, we will assume $H \geqq 0$. We are interested in obtaining information about the Gauss curvature $K$ of $S$ at the origin. In the minimal surface case $H=0$, Heinz [4] showed that, for all such surfaces $S$, one has $|K| \leqq c / R^{2}, c=4 \pi^{3} / 3$.

In [3], Finn and Osserman gave a completely different method for obtaining such a bound. They also showed that if $S$ has a horizontal tangent plane at the origin, then the best possible value of $c$ is $\pi^{2} / 2$, with the classical Scherk's surface playing the role of an extremal.

Recall that Scherk's surface is defined by the expression

$$
z=(\sqrt{ } 2 R / \pi)(\log \cos (\pi y / \sqrt{ } 2 R)-\log \cos (\pi x / \sqrt{ } 2 R))
$$

over the square $|x|<R / \sqrt{ } 2,|y|<R / \sqrt{ } 2$, and takes on the boundary values $\pm \infty$, on alternate sides of the square. Recently the author [9] has proven the existence of a "Scherk type" surface of constant mean curvature

Received by the editors November 24, 1971.

AMS 1970 subject classifications. Primary 53A10; Secondary 35B45, 35G30.

Key words and phrases. Constant mean curvature, minimal surfaces, Gauss curvature, a priori estimate, Scherk surface, comparison methods, maximum principle.

${ }^{1}$ Research supported in part by NSF Grant GU-2582.

c) American Mathematical Society 1972 
exhibiting the same general behavior as the classical Scherk's surface. The domain $D$ of definition of this unusual surface may be described as follows. For any $R \leqq 1 / 2 H, D$ is a domain contained in $x^{2}+y^{2} \leqq R^{2}$ which is bounded by two circular arcs of curvature $+2 \mathrm{H}$ and two circular arcs of curvature $-2 H$, and is symmetric about the $x$ and $y$ axes. The situation is illustrated in Figure 1. The solution takes on the boundary values $+\infty$ on the convex

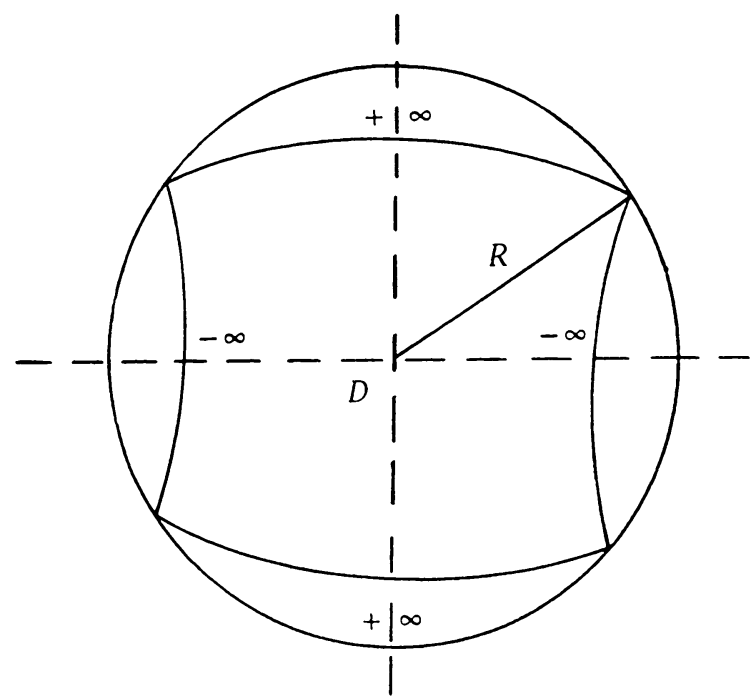

FIGURE 1

circular arcs and $-\infty$ on the concave circular arcs. The exact geometry of the configuration is determined by the relationship

$$
l^{+}-l^{-}=2 H(\text { area of } D)
$$

where $l^{+}$is the length of the convex arcs and $l^{-}$is the length of the concave aics. As $H \rightarrow 0$ the circular arcs become straight and $D$ tends to the square over which the classical Scherk's surface is defined.

The main result of this paper is that the Gauss curvature of our Scherk type surface provides a sharp bound for the Gauss curvature at the origin for all solutions of (1) defined over $x^{2}+y^{2} \leqq R^{2} \leqq 1 / 4 H^{2}$ with horizontal tangent plane at the origin. As in the minimal surface case equality is never attained although there is a sequence of solutions defined over $x^{2}+y^{2} \leqq R^{2}$ whose curvature at the origin approaches the extremal bound. A precise statement of our main result is given in Theorem 1. The method of proof will be a modification of the comparison method used by Finn and Osserman. 
The author is indebted to Professor Robert Finn for helpful conversations.

\section{Preliminary results.}

Notation. For each $R \leqq 1 / 2 H$ we will denote by $v(R ; x, y)$ the Scherk type surface of constant mean curvature $H>0$ that corresponds to the disc of radius $R$, as described in the introduction. Also we will denote by $K(R)$ the Gauss curvature of $v$ at the origin.

The following useful lemma is well known [8].

LEMMA 1. Let $u$ and $v$ be solutions of (1) in a neighborhood of the origin 0 . Suppose that $u \neq v$ while $\nabla u=\nabla v$ at 0 . Then for some integer $k \geqq 2$ we have $v-u=$ constant $+P(\bar{x}, \bar{y})+O\left(r^{k+1}\right), r=\left(x^{2}+y^{2}\right)^{1 / 2}$ where $P(\bar{x}, \bar{y})$ is a harmonic (homogeneous) polynomial of degree $k$ in the variables $\bar{x}, \bar{y}$, and $\bar{x}, \bar{y}$ are affinely related to $x, y$.

It follows that a neighborhood of 0 is subdivided by $k \geqq 2$ smooth curves into $2 k \geqq 4$ sectors in such a way that $u-v$ alternates sign in each sector.

We next discuss an "explicit" solution of (1) which is needed in later results. If we look for a solution of (1) of the form $u=u(r)$, then we are led to the ordinary differential equation

$$
u^{\prime} /\left(1+u^{\prime 2}\right)^{1 / 2}=H r+A r^{-1}
$$

where $A$ is an arbitrary constant. In the case $A>0$, no solution exists unless $4 A H<1$. If this condition is satisfied there is a solution $w(r)$ defined in the annulus

$$
\left(1-(1-4 A H)^{1 / 2}\right) / 2 H<r<\left(1+(1-4 A H)^{1 / 2}\right) / 2 H .
$$

This annulus is contained in the disc of radius $1 / H$ and is symmetric about the circle of radius $1 / 2 \mathrm{H}$. On the inner circumference the exterior normal derivative $\partial u / \partial n=-\infty$ while on outer circumference $\partial u / \partial n=+\infty$.

In the following discussion we will use the notation $w\left(R_{1}, r\right)$ to indicate that $R_{1}$ is the inner radius of the annulus of definition of $w(r)$ defined by (3).

LEMMA 2. The surfaces $v(R ; x, y)$ satisfy

(i) $v(R ; x, y)=v(R ; x,-y)=v(R ;-x, y)$ so that

$$
v_{x}(R ; 0,0)=v_{y}(R ; 0,0)=v_{x y}(R ; 0, y)=v_{x y}(R ; x, 0)=0 .
$$

(ii) $K(R)<2 R H-1 / R^{2}$ so that $K(R) \rightarrow-\infty$ as $R \rightarrow 0$.

Proof. (i) Observe that each of the functions $v(R ; x, y), v(R ; x,-y)$, $v(R ;-x, y)$ are solutions of $(1)$, have the same boundary data and agree at the origin. Hence by the uniqueness theorem established in $[9$, Theorem 7.1] they must be identical. The conclusions of (i) now follow immediately. 
(ii) Consider the surface of revolution of constant mean curvature $H$ that corresponds to the solution $w(, r)$ discussed above. In 1841, Delaunay [1] found that the generating curve of this surface of revolution is cbtained by tracing out the path of the focus of an ellipse as the ellipse rolls without slipping along the axis of the surface. Using the notation of (3), the ellipse that corresponds to $w(, r)$ will have a major semiaxis of length $1 / 2 H$ and a minor semiaxis of length $(A / H)^{1 / 2}$ where $0<A<1 / 4 H$. The generating curve is illustrated in Figure 2. If we revolve the generating curve about the

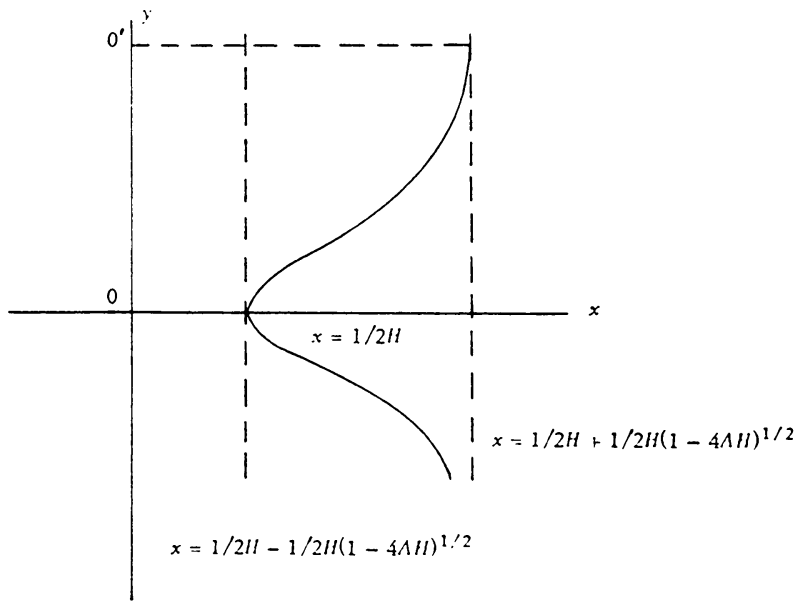

FIGURE 2

$y$-axis and consider the "bottom" half of the surface cut off by the $x$ - $y$ plane, then we have a surface of constant mean curvature $+H$ defined over the symmetric domain $\Delta$ in the plane defined by the generating curve and its reflection about the $y$-axis. We have oriented things so that the origin 0 lies on a "waist" of this surface. Let us denote this surface by $z=u(x, y)$. Evidently $\nabla u(0)=\nabla u\left(0^{\prime}\right)=0$ and $u_{x y}=0$ on the $y$ axis. (Note that the point $0^{\prime}$ corresponds to an "outer waist" of the surface.) Using the formula $K=w^{\prime} w^{\prime \prime} / r\left(1+w^{\prime 2}\right)^{2}$, a simple computation using (2) shows that $K=H^{2}-A^{2} / r^{4}$ where $r$ is the distance from a point on the generating curve to the $y$-axis. We now choose $A=R(1-R H)$. Then the Gauss curvature $K(0)$ of the surface $z=u(x, y)$ at 0 is equal to $(2 R H-1) / R^{2}$. With this choice of $A$, the disc of radius $R$ in which $v(R ; x, y)$ is defined is just contained in $\Delta$. The proof of part (ii) now amounts to showing that $K(R)<$ $K(0)$. To do this, suppose for the moment that $K(R)<0$; then if $0>$ $K(R) \geqq K(0)$ we can let the parameter $A$ increase towards its maximum value of $1 / 4 H$. This has the effect of decreasing $|K(0)|$ to the value zero while the domain $\Delta$ becomes more and more like a strip of width $1 / H$. We stop this 
process when $K(R)=K(0)$. We can then rotate the surface $v(R ; x, y)$ (i.e. use $v(R ; y, x)$ if necessary) so that the second derivatives of $u$ and $v(R ; x, y)$ coincide at the origin. By Lemma 1 there are at least six level curves of $\varphi(x, y)=u(x, y)-v(R ; x, y)$ passing through the origin and dividing a neighborhood of the origin into sectors such that $\varphi$ alternates sign in each sector. Consider any component $U$ of the open set $\{\varphi \neq 0\} \cap D$, where $D$ is the domain of definition of $v(R ; x, y)$. At an interior boundary point of $U, \varphi=0$ so that by the maximum principle for differences of solutions of (1), the boundary of $U$ cannot consist entirely of interior points of $D$. But if the boundary of $U$ contains an "inner point" of a side of $D$ then it must contain the whole side. Hence the set $\{\varphi \neq 0\} \cap D$ has at most four components whose boundaries contain inner points of sides of $D$. On the other hand, the set $\{\varphi \neq 0\} \cap D$ has at least six components since this is true near the origin and otherwise some component would have all its boundary points interior to $D$ which was seen to be impossible. Hence some component $U$ of $\{\varphi \neq 0\} \cap D$ has as boundary points only interior points of $D$ and one or more "vertices" of $D$. By the general maximum principle [9] this is impossible. Thus the assumption $K(R) \geqq$ $(2 R H-1) / R^{2}$ leads to a contradiction once we know that $K(R)<0$. So suppose $K(R) \geqq 0$. Translate the surface $z=v(R ; x, y)$ along the positive $y$-axis so that it is centered at the point $0^{\prime}$ of Figure 2 . At $0^{\prime}$ the value of the Gauss curvature of the surface $z=u(x, y)$ achieves its maximum value. If $K(R)$ is less than this value we again let $A$ tend to $1 / 4 H$. Then this maximum curvature tends to the value 0 so that at some point the curvatures of the two surfaces become equal. A repetition of the preceding argument gives a contradiction. If $K(R)$ is greater than this maximum value we decrease $A$ to the value zero. This has the effect of increasing the maximum curvature of $z=u(x, y)$ to the value $H^{2}$ while the domain $\Delta$ becomes more and more like a sequence of circles of radius $1 / H$ stacked on top of each other. Thus at some point we can reach a situation in which the two surfaces have the same Gauss curvature and the same domain of definition. Hence we must have $K(R)<0$ proving the lemma.

LEMMA 3. The values $K(R)$ depend continuously on $R$.

Proof. We normalize $v(R ; x, y)$ so that $v(R ; 0,0)=0$. Consider a fixed $R_{0} \leqq 1 / 2 H$ and a sequence $\left\{R_{n}\right\}$ converging to $R_{0}$ with $R_{n} \leqq 1 / 2 H$. Let $D(R)$ be the domain of $v(R ;)$. Then the corresponding domains $D\left(R_{n}\right)$ converge to $D\left(R_{0}\right)$. According to the proof of Theorem 6.4 of [9] the sequence $\left\{v\left(R_{n} ;\right)\right\}$ must be uniformly bounded on compact subsets of $D\left(R_{0}\right)$. Hence by the compactness principle [8, Theorem 3$]$ a subsequence of $\left\{v\left(R_{n} ;\right)\right\}$ must converge to a solution $v(x, y)$ of $(1)$ in $D\left(R_{0}\right)$. We claim $v(x, y)$ must equal $v\left(R_{0} ; x, y\right)$. To see this, note that any point near the 
boundary of $D\left(R_{0}\right)$ is eventually near the boundary of $D\left(R_{n}\right)$. It then follows from the proof of Theorem 6.4 of [9] that $v(x, y)$ has the same boundary values as $v\left(R_{0} ; x, y\right)$. By Theorem 7.1 of [9], $v\left(R_{0} ;\right)$ and $v$ differ by a constant which must be zero by normalization. Since the above argument holds for any subsequence of the original sequence, it follows that the entire sequence $\left\{v\left(R_{n} ;\right)\right\}$ converges to $v\left(R_{0} ;\right)$. Now a bound on the sequence $\left\{v\left(R_{n} ;\right)\right\}$ in a neighborhood of the origin implies a uniform bound on $\left|\nabla v\left(R_{n} ;\right)\right|$ in a neighborhood of the origin [8]. Consequently we can think of the $v\left(R_{n} ;\right)$ as solutions of a uniformly elliptic equation with a fixed modulus of ellipticity. This implies that $\nabla v\left(R_{n} ;\right)$ are uniformly Hölder continuous [5] in a still smaller neighborhood of the origin. The classical Schauder estimates show that the second derivatives of the sequence are also uniformly Hölder continuous in this neighborhood, so that by the Arzela-Ascoli theorem, the first and second derivatives of $\left\{v\left(R_{n} ;\right)\right\}$ at the origin converge uniformly to those of $v\left(R_{0} ;\right)$. Hence $\left\{K\left(R_{n}\right)\right\}$ converges to $K\left(R_{0}\right)$ proving the lemma.

\section{The main result; final remarks.}

THEOREM 1. Let $z=u(x, y)$ be a surface of constant mean curvature $H$ defined over the disc $x^{2}+y^{2}<R_{0}^{2}$ with $\nabla u(0,0)=0$. Then, for $R_{0} \leqq 1 / 2 H$,

$$
H^{2} \geqq K>K\left(R_{0}\right)
$$

at the origin. Moreover $K\left(R_{0}\right)<\left(2 R_{0} H-1\right) / R_{0}^{2}$ and the lower bound $K\left(R_{0}\right)$ is never actually attained although there is a sequence of solutions defined over $x^{2}+y^{2}<R_{0}^{2}$ whose curvature at the origin approaches $K\left(R_{0}\right)$.

REMARK. Of course if $R_{0}>1 / 2 H$ we still have an a priori estimate for $K$ by restricting $u$ to the disc of radius $1 / 2 H$.

Proof. The inequality $K \leqq H^{2}$ is always satisfied for any surface so that we need only prove the right-hand side of (3). Suppose that $K \leqq K\left(R_{0}\right)$. By Lemma $2, K(R)$ tends to $-\infty$ as $R \rightarrow 0$. By Lemma 3 we can choose $R$ such that $K=K(R)$. Then by rotating one of the surfaces, we can make all second derivatives of $u$ and $v(R ;)$ coincide at the origin. Thus $u$ and $v(R ;)$ have a "second order contact" at the origin (by a translation we may assume $u(0,0)=v(R ; 0,0))$. By the argument already given in the proof of Lemma 2 , this is impossible. By the proof of the existence of $v\left(R_{0} ;\right)$ given in [9], there is a sequence of solutions defined over $x^{2}+y^{2}<R_{0}^{2}$ converging to $v\left(R_{0} ;\right)$. A repetition of the argument of Lemma 3 shows that their curvatures at the origin tend to $K\left(R_{0}\right)$. This proves the theorem.

FINAL REMARKS. It seems quite likely that a priori bounds for the Gauss curvature at the origin exist independent of the slope of the solution surface at the origin. By considering Scherk type comparison surfaces with 
less symmetry it is possible to improve Theorem 1 somewhat. However it is not at all clear whether the comparison method could be used to prove a general result.

Another interesting point is that solutions of (1) in general do not exist for $R$ in the range $1 / 2 H<R \leqq 1 / H$. That is, boundary data cannot be prescribed arbitrarily. We expect that solutions of (1) in this range must be subject to severe a priori limitations. For example, R. Finn has conjectured that the oscillation of all such solutions must be uniformly bounded. We also suspect strong limitations on the Gauss curvature of such surfaces.

\section{REFERENCES}

1. Ch. Delaunay, Sur la surface de revolution dont la courbure moyenne est constante J. Math. Pures Appl. 6 (1841), 309-315.

2. R. Finn, Remarks relevant to minimal surfaces, and to surfaces of prescribed mean curvature, J. Analyse Math. 14 (1965), 139-160. MR 32 \#6337.

3. R. Finn and R. Osserman, On the Gauss curvature of nonparametric minimal surfaces, J. Analyse Math. 12 (1964), 351-364. MR 29 \#3967.

4. E. Heinz, Über die Lösungen der Minimalfächengleichung, Nachr. Akad. Wiss. Göttingen. Math. Phys. Kl. Math.-Phys.-Chem. Abt. 1952, 51-56. MR 14, 885.

5. L. Nirenberg, On nonlinear elliptic partial differential equations and Hölder continuity, Comm. Pure Appl. Math. 6 (1952), 103-156; Addendum, 395.

6. J. C. C. Nitsche, On new results in the theory of minimal surfaces, Bull. Amer. Math. Soc. 71 (1965), 195-270. MR 30 \#4200.

7. R. Osserman, A survey of minimal surfaces, Van Nostrand Reinhold, New York, 1969. MR 41 \#934.

8. J. Serrin, The Dirichlet problem for surfaces of constant mean curvature, Proc. London Math. Soc. (3) 21 (1970), 361-384. MR 43 \#1093.

9. J. Spruck, Infinite boundary value problems for surfaces of constant mean curvature, Arch. Rational Mech. Anal. (to appear).

Department of Mathematics, University of New Mexico, Albuquerque, New MEXICO 87106 